\title{
Mixoma cardiaco: Caracterización clínica, métodos diagnósticos y resultados alejados del tratamiento quirúrgico durante tres décadas de experiencia
}

\author{
Pedro Becker $\mathbf{R}^{1}$, Alejandro Ramírez $\mathbf{M}^{\mathrm{a}}$, Ricardo Zalaquett $\mathbf{S}^{\mathbf{1}}$, \\ Sergio Morán $\mathbf{V}^{1}$, Manuel J Irarrázaval $\mathbf{L I}^{1}$, Claudio Arretz $\mathbf{V}^{\mathbf{1}}$, \\ Samuel Córdova $\mathbf{A}^{\mathbf{1}}$, Pilar Arnaiz $\mathbf{G}^{2}$. \\ Cardiac myxoma: \\ Clinical characterization, diagnostic \\ methods and late surgical results
}

Background: Cardiac myxoma is the most common primary cardiac tumor. Aim: To evaluate clinical aspects, diagnostic methods and surgical outcomes in patients with cardiac myxoma. Patients and Methods: All patients who underwent surgical resection of a cardiac myxoma between January 1973 and December 2004 at our institution, were identified and their medical records and diagnostic data reviewed. Results: Thirty seven patients identified (24 women), with a median age of 54 years (range 2-74). The most common symptom was dyspnea (51\%), followed by embolism (24\%) and cardiac murmurs (16\%). The diagnosis was made by echocardiogram in $84 \%$ of patients. The mean tumor size was $4.9 \pm 1.3 \mathrm{~cm}$. The tumor was located in the left atrium in $83 \%$ of the patients, right atrium in $8 \%$ and left ventricle in $3 \%$. Two patients had multiple tumors. Surgical approach was transeptal in $51 \%$, left atriotomy in $22 \%$, combined transeptal and roof of the left atrium in 19\% and right atriotomy in $8 \%$. The tumor and its attachment base were excised in one piece. There were two operative deaths and five late deaths. The mean follow-up was 11.5 years. The 5, 10, 15 and 20 years survival was $89 \%, 83 \%, 75 \%$ and $64 \%$, respectively. Two patients had recurrence (5.4\%), at 2 and 25 years after surgery, respectively, and the recurrence free survival at 10 and 25 years was $97 \%$ and $73 \%$, respectively. Conclusions: Dyspnea was the most common symptom and echocardiogram the main diagnostic method. Surgical treatment was highly effective, with low mortality and recurrence rate, with good long term survival (Rev Méd Chile 2008; 136: 287-95).

(Key w ords: Echocardiography; Heart neoplasms; Myxoma)

\footnotetext{
Recibido el 18 de julio, 2007. Aceptado el 28 de septiembre, 2007.

${ }^{1}$ Departamento de Enfermedades Cardiovasculares. ${ }^{2}$ Departamento de Pediatría, Pontificia Universidad Católica de Chile. Santiago, Chile.

${ }^{a}$ Interno de la Facultad de Medicina de la Pontificia Universidad Católica de Chile.
}

Correspondencia a: Dr. Pedro Becker Rencoret. Marcoleta 367, 6o piso. Santiago, Chile. Teléfono: 562633 3030. Fax: 562639 0108. E mail: pbecker@med.puc.cl 
$\mathrm{L}$ os tumores del corazón corresponden mayoritariamente a metástasis, siendo los tumores primarios menos frecuentes. Se estima que éstos últimos tienen una incidencia entre 0,0017\% y $0,05 \%$ en autopsias. El mixoma cardiaco corresponde al tumor cardiaco primario más frecuente, constituyendo entre 30\% y 50\% en distintas series de tumores primarios que afectan dicho órgano. Su localización más frecuente es la aurícula izquierda ${ }^{1,2}$. Tiene aspecto gelatinoideo y mamelonado (Figura 1a); frecuentemente es un tumor pedunculado pero puede tener una base ancha de implantación. Histológicamente se caracteriza por estar revestido de endotelio y por la presencia de células poligonales inmersas en una matriz de mucopolisacáridos (Figura 1b) ${ }^{3}$. Aunque es un tumor benigno, algunos pueden recurrir e incluso metastizar ${ }^{4,5}$. Debido a su localización, puede generar obstrucción al flujo de sangre dentro del corazón o embolizar, siendo por lo tanto potencialmente letal $^{6,7}$.

Nuestro grupo comunicó sus primeros 8 casos operados durante la década 1970-79, con muy buenos resultados precoces ${ }^{8}$. El interés del presente estudio es revisar la experiencia acumulada durante tres décadas en el tratamiento quirúrgico de pacientes portadores de mixoma cardiaco en nuestra institución, con el objetivo de describir sus características clínicas, métodos de estudio, técnicas quirúrgicas, resultados inmediatos y alejados.

\section{PACIENTES Y MÉTODO}

Se identificaron todos los pacientes sometidos consecutivamente a resección de mixoma cardiaco entre enero de 1973 y diciembre de 2004, en la base de datos del Servicio de Cirugía Cardiovascular. El estudio se cerró en marzo de 2006, de modo que los pacientes tuvieran al menos un año de seguimiento. Se analizaron retrospectivamente sus antecedentes clínicos, protocolos operatorios, ecocardiografías, angiografías y estudios anatomopatológicos. Se actualizó el seguimiento mediante evaluación clínica y ecocardiográfica.

La edad y tiempo de seguimiento se expresan en mediana y sus rangos. El tamaño del tumor y gradiente transmitral se expresan en promedio y desviación estándar. Se calculó la sobrevida alejada según análisis de Kaplan-Meier.

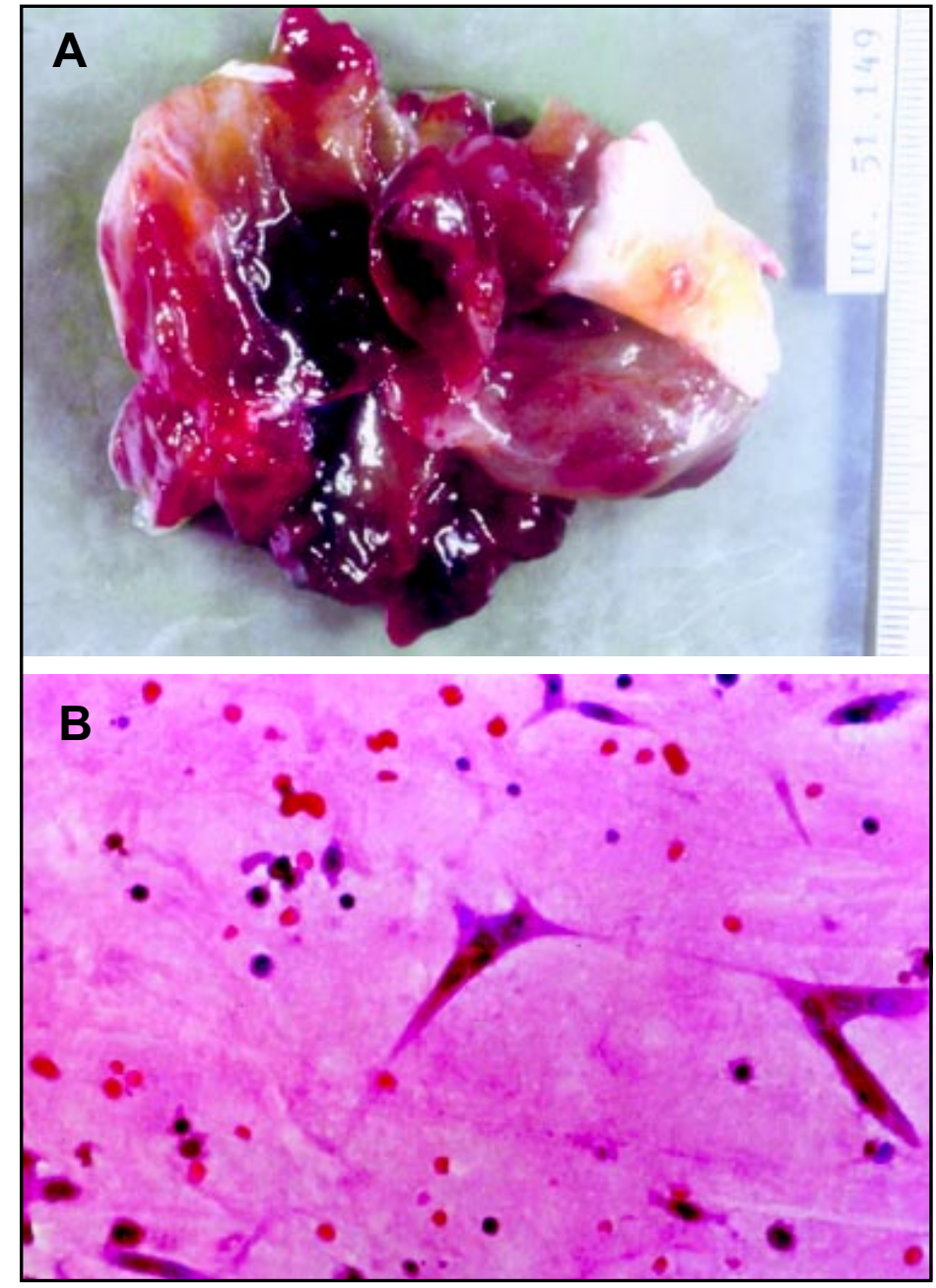

Figura 1. a. Mixoma cardiaco auricular izquierdo. b. Histología caracterizada por células poligonales inmersas en matriz de mucopolisacáridos. 


\section{RESUlTADOS}

La serie se compone de 37 pacientes, 24 mujeres y 13 hombres, con edad de 53,5 años (2 a 74).

La forma de presentación clínica más frecuente fue la disnea, seguida de la embolia sistémica; con menor frecuencia lo fue el hallazgo de soplo cardiaco aislado. Tres pacientes fueron asintomáticos (Tabla 1).

El diagnóstico se realizó por ecocardiografía en 29 pacientes (Figura 2). En un paciente el diagnóstico se sospechó por un TAC de tórax con imagen sugerente, y en otro por una ecografía abdominal. En ambos se confirmó el diagnóstico por ecocardiografía. En la era preecocardiográfica, 5 pacientes tuvieron diagnóstico angiográfico. En un paciente fue hallazgo intraoperatorio (Tabla 2).
En 83\% de los casos, el mixoma se ubicó en la aurícula izquierda, implantado mayoritariamente en el septum interauricular. El resto se ubicó en la aurícula derecha, biauricular y ventrículo derecho, aurícula izquierda y ventrículo izquierdo (Tabla 3).

Técnica quirúrgica. Todos los pacientes fueron operados con circulación extracorpórea e hipotermia moderada. A partir del año 1977 se empleó en todos cardioplejia cristaloide. El abordaje quirúrgico fue durante los primeros años de la serie a través de la aurícula izquierda, mediante incisión por detrás del surco interauricular. Posteriormente se hizo más frecuente el abordaje transeptal exclusivo a través de la aurícula derecha (Figura 3), o con extensión al techo de la aurícula izquierda (transeptal y superior combinado), lo que coincidió

Tabla 1. Forma de presentación clínica predominante en pacientes con mixoma cardiaco

\begin{tabular}{|lrc|}
\hline Presentación clínica & № de pacientes (\%) \\
\hline Disnea & 19 & $(51,4)$ \\
Embolia sistémica & 9 & $(24,3)$ \\
Soplo cardiaco & 6 & $(16,2)$ \\
Asintomático & 3 & $(8,1)$ \\
\hline
\end{tabular}

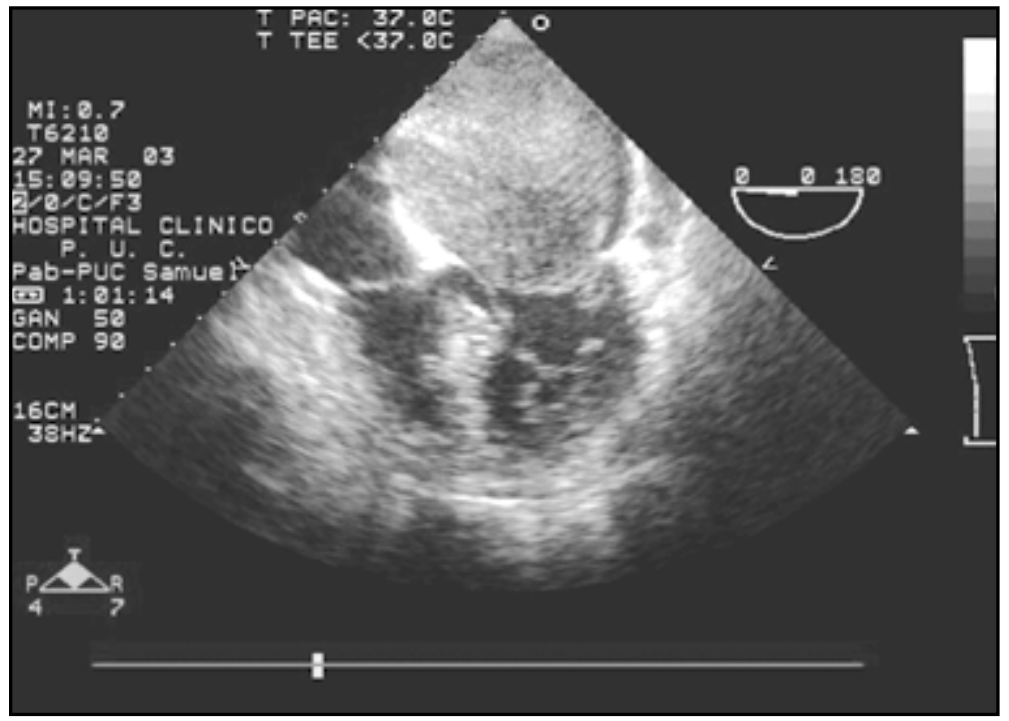

Figura 2. Ecocardiograma sugerente de mixoma cardiaco ocupando prácticamente toda la aurícula izquierda. 
Tabla 2. M étodo diagnóstico de mixoma cardiaco.

Considera forma de realizar diagnóstico previo a resultado de biopsia

\begin{tabular}{|lrc|}
\hline Método diagnóstico & № de pacientes (\%) \\
\hline Ecocardiografía & 31 & $(83,8)$ \\
Angiografía & 5 & $(13,5)$ \\
Hallazgo intraoperatorio & 1 & $(2,7)$ \\
\hline
\end{tabular}

Tabla 3. U bicación del mixoma cardiaco

\begin{tabular}{|lcc|}
\hline Ubicación & № de pacientes (\%) \\
\hline Aurícula izquierda & 31 & $(83)$ \\
Aurícula derecha & 3 & $(8)$ \\
Biauricular & 1 & $(3)$ \\
Aurícula izquierdo y ventrículo izquierdo & 1 & $(3)$ \\
Ventrículo izquierdo & 1 & $(3)$ \\
\hline
\end{tabular}

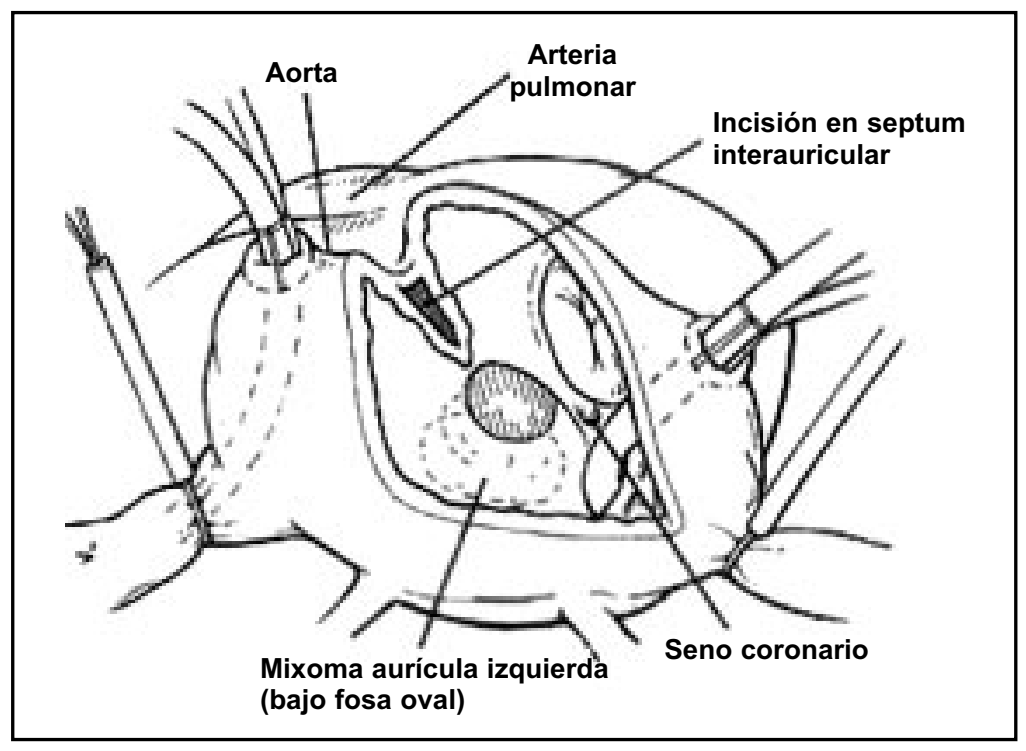

Figura 3. Resección de mixoma auricular izquierdo por vía transeptal.

con la adopción de estas técnicas como uso preferente en la cirugía mitral durante las últimas dos décadas? ${ }^{9}$. De los 7 pacientes que requirieron un abordaje transeptal y superior combinado, uno requirió además abordaje transaórtico por componente tumoral ventricular izquierdo.
En 15 pacientes se utilizó parche para reparar el defecto interauricular, de los cuales, 14 recibieron parche de pericardio autólogo y uno parche de politetrafluoretileno (PTFE). En el resto se realizó cierre directo del defecto interauricular creado. Dos pacientes fueron operados de emer- 
gencia por compromiso hemodinámico grave. En todos se confirmó el diagnóstico por estudio anatomopatológico (Tabla 4).

Resultados quirúrgicos. Mortalidad operatoria: Dos pacientes fallecieron en el posoperatorio inmediato $(5,4 \%)$. La primera paciente, operada en shock cardiogénico, salió de circulación extracorpórea en condiciones marginales y falleció en pabellón (1977). La segunda paciente desarrolló en el posoperatorio inmediato un síndrome vasopléjico seguido de shock irreversible y falla multiorgánica, falleciendo a las $72 \mathrm{~h}$. La autopsia no logró explicar la causa originaria (1998). Ambas pacientes eran portadoras de mixoma de aurícula izquierda.

El seguimiento fue de 138 meses (14 a 407). Cinco pacientes fallecieron alejadamente por causas no relacionadas al mixoma.

Dos pacientes presentaron recurrencia del mixoma (5,4\%). El primer caso corresponde a un hombre de 24 años operado de un mixoma biauricular, quien presentó una recurrencia en el ventrículo derecho (septum) 25 años después, siendo reoperado exitosamente. El segundo caso corresponde a un niño de dos años operado por un mixoma auricular izquierdo, quien presentó una recurrencia en aurícula izquierda a los 2 años cuatro meses de operado, siendo también exitosamente reintervenido sin recurrencia luego de 3 años de seguimiento. Por lo tanto, $75,7 \%$ de la serie original están vivos y sin evidencias de recurrencia, siendo la sobrevida libre de recurrencia a 10 años de 97\% (Figura 4), y la sobrevida actuarial a 10 años de $83,1 \%$ (Figura 5).

\section{DisCUSIÓN}

A pesar de ser el mixoma cardiaco el tumor primario más frecuente de corazón, pocos estudios en la literatura comunican seguimientos alejados y con un número importante de casos. En este estudio reportamos nuestra experiencia por más de treinta años en el manejo de esta patología, poniendo énfasis en los resultados del tratamiento quirúrgico, constituyendo la serie más numerosa y con más años de seguimiento reportada en nuestro medio.

Las formas de presentación del mixoma cardiaco son diversas. Por un lado existen aquellas derivadas de su efecto mecánico, ya sea presentándose sólo como un soplo cardiaco o como insuficiencia cardiaca congestiva con grados variables de disnea, como ocurre en muchos casos de mixomas de aurícula izquierda que se comportan como una estenosis mitral severa. Fragmentos del tumor o trombos intracavitarios alrededor de éste pueden embolizar a la circulación sistémica, así como también al árbol vascular pulmonar, en los casos de mixomas ubicados en las cavidades derechas ${ }^{6,7}$. Con la acumulación de casos y experiencia, se ha hecho evidente cómo algunos mixomas pueden tener presentaciones atípicas Tabla 4. Tratamiento quirúrgico del mixoma cardiaco. Abordaje quirúrgico, forma de reparación
del septum interauricular, cirugías realizadas con carácter de emergencia

\begin{tabular}{|c|c|c|}
\hline \multirow{2}{*}{$\begin{array}{l}\text { Abordaje quirúrgico } \\
\text { Transeptal }\end{array}$} & \multicolumn{2}{|c|}{ № de pacientes (\%) } \\
\hline & 19 & (51) \\
\hline Auricular izquierdo & 8 & (22) \\
\hline Transeptal y superior combinado * & 7 & (19) \\
\hline Auricular derecho & 3 & (8) \\
\hline \multicolumn{3}{|l|}{ Reparación } \\
\hline Cierre con parche & 15 & (41) \\
\hline Cierre directo & 22 & (59) \\
\hline Emergencia & 2 & $(5,4)$ \\
\hline
\end{tabular}

*En este grupo un paciente además requirió abordaje transaórtico. 


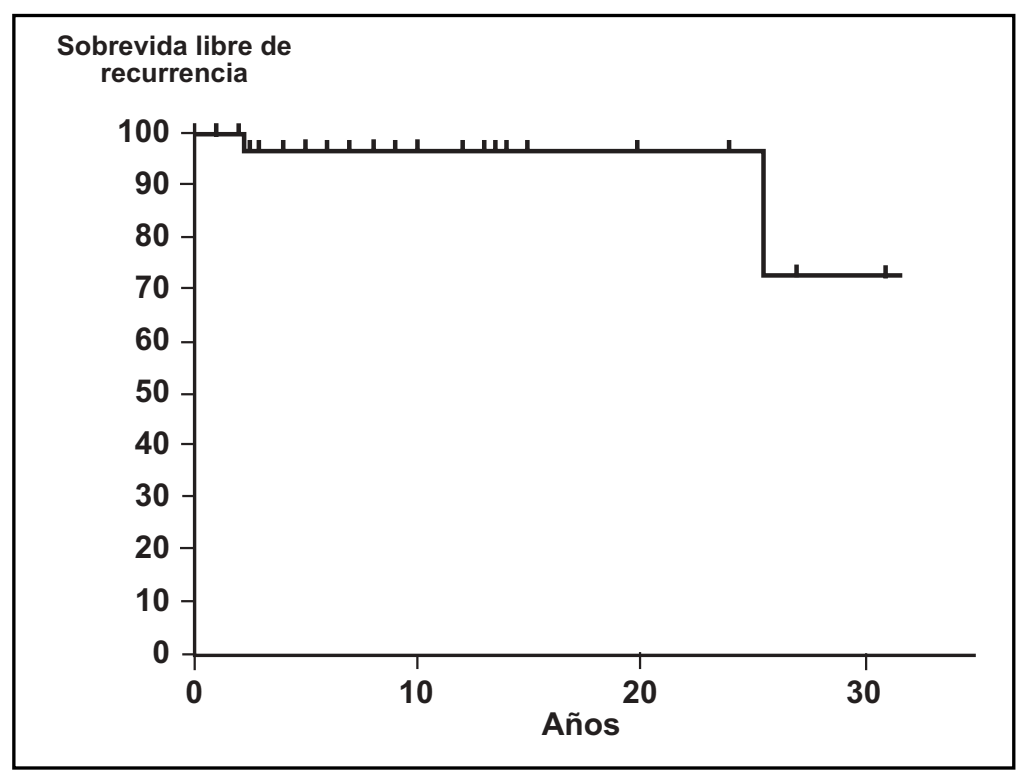

Figura 4. Curva de Kaplan Meier, muestra la sobrevida libre de recurrencia después del tratamiento quirúrgico del mixoma cardíaco. Sobrevida libre de recurrencia a 10 años: 0,97; a 25 años: 0,73.

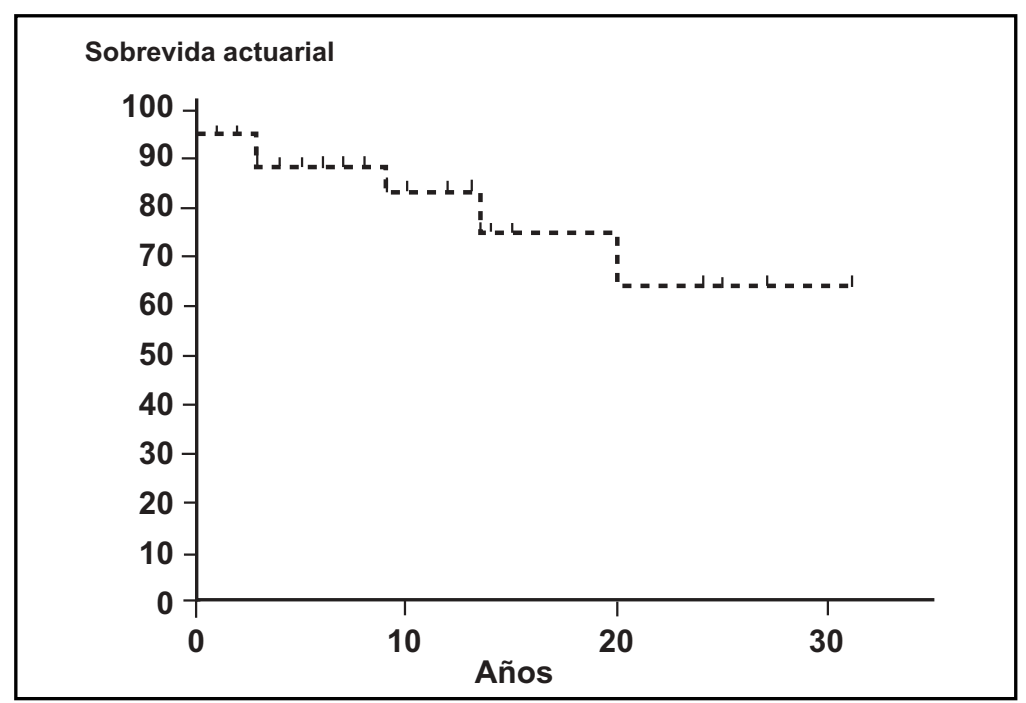

Figura 5. Curva de Kaplan Meier, muestra la sobrevida actuarial después del tratamiento quirúrgico del mixoma cardíaco. Sobrevida a 5 años: 0,89; 10 años: 0,83; 15 años: 0,75; 20 años: 0,64.

tales como fatiga, fiebre, eritema, artralgia, mialgias, pérdida de peso, anemia, elevaciones de la proteína C reactiva y de la $\mathrm{VHS}^{10-13}$, derivadas de la producción de interleukinas proinflamatorias, como la IL-6 ${ }^{14,15}$. Un factor importante en la pesquisa de pacientes asintomáticos lo constituye el uso cada vez más frecuente de la ecocardiografía, que con sus avances nos permite hoy en día acercarnos al diagnóstico y tener un visión preoperatoria que nos ayuda a planificar un 
adecuado abordaje quirúrgico ${ }^{16-20}$. En nuestra serie, al igual que en otros reportes en la literatura, el principal síntoma de presentación fue la disnea, seguido por la embolia y el hallazgo de un soplo cardiaco. El diagnóstico se realizó en la gran mayoría de los casos por ecocardiografía, salvo aquellos pacientes de la serie en que el diagnóstico se hizo mediante estudio angiográfico, en la era preecocardiográfica.

El sitio más frecuente de ubicación del mixoma cardiaco fue la aurícula izquierda, especialmente en relación al septum interauricular. Esta ubicación, bastante constante en los casos esporádicos de mixoma cardiaco, ha generado diversas hipótesis sobre el origen de estas células tumorales, ninguna de las cuales ha sido probada fehacientemente como la causa del mixoma cardiaco $^{21}$.

Desde que Crafoord, en la década de 1950-59, lograra por primera vez la resección exitosa de un mixoma cardiaco ${ }^{22}$, diversos avances, tanto en la medicina como en la cardiocirugía, han permitido disminuir la morbimortalidad asociada a dicho procedimiento, constituyéndose hoy en día en el tratamiento de elección. Una vez establecido el diagnóstico, la cirugía debe realizarse prontamente, disminuyendo así la posibilidad de embolia o muerte súbita ${ }^{23,24}$. El abordaje quirúrgico para el mixoma de aurícula izquierda se ha realizado en general de tres formas: transeptal por atriotomía derecha, por acceso transeptal y superior combinado y por atriotomía izquierda. Si bien en muchos centros las dos primeras se han preferido con bastante frecuencia, el abordaje óptimo es aún tema de discusión ${ }^{25-28}$. En nuestra serie, el abordaje más utilizado fue el transeptal, siendo en los últimos años el abordaje transeptal y superior combinado la vía de elección, gracias a que permite una excelente exposición, haciendo técnicamente más fácil la resección en una pieza del tumor junto a su base de implantación en el septum interauricular (Figura 1a).

\section{REFERENCIAS}

1. Shapiro L. Cardiac tumours: diagnosis and management. Heart 2001; 85: 218-22.

2. Bukley GH, Hutchins GM. Atrial myxomas: a fifty years review. Am Heart J 1979; 97: 639-43.
La recurrencia de los mixomas cardiacos comunicada en varias publicaciones se acerca a 5\%. Factores que se han asociado a esta recurrencia son, entre otros, una incompleta resección quirúrgica, la transmisión autosómica dominante de algunos mixomas cardiacos, como ocurre en el síndrome de Carney, caracterizado por presentar además lesiones lentiginosas en la piel y otros tumores, y la presencia de aneuploidía en las células tumorales ${ }^{29-34}$. Ninguno de los dos pacientes que presentaron recurrencia en esta serie corresponden a mixoma familiar o síndrome de Carney. En el paciente pediátrico que recurrió a los 2 años se hizo estudio del ADN tumoral, descartándose aneuploidia celular ${ }^{35}$

Sólo identificamos un caso de mixoma familiar, el cual se ha mantenido libre de recurrencia.

La mortalidad perioperatoria en nuestra experiencia ha sido comparable a la de otras series. El primer caso fatal, operado de extrema urgencia en los albores de esta serie, ponen de manifiesto la importancia del diagnóstico y tratamiento oportuno, siendo ésta una condición que requiere solución quirúrgica relativamente urgente. En el segundo caso, no encontramos una explicación vinculada al mixoma propiamente tal que explicara el desarrollo de síndrome vasopléjico seguido de falla multiorgánica y muerte. Las muertes alejadas no tuvieron relación con el mixoma.

Podemos concluir que el tratamiento quirúrgico de pacientes con mixoma cardiaco ha sido altamente efectivo en nuestra experiencia, con sobrevida alejada que asemeja curvas se sobrevida de la población general y resultados comparables a series de otros autores ${ }^{28,36-38}$.

\section{Agradecimientos}

Nuestro reconocimiento y agradecimiento a los doctores Juan Dubernet y Gustavo Maturana quienes operaron varios pacientes durante la primera etapa de esta serie y estimularon esta revisión clínica.

3. Pucci A, Gagliardotto P, Zanini C, Pansini S, Di Summa M, Mollo F. Histopathologic and clinical characterization of cardiac myxoma: review of 53 cases from a single institution. Am Heart J 2000; 140: 134-8.

4. Read RC, White HJ, Murphy ML, Williams D, Sun SN, Flanagan WH. The malignant potentiality of 
left atrial myxoma. J Thorac Cardiovasc Surg 1974; 68: 857-67.

5. Kaynak K, Besirli K, Arslan C, Ozguroglu M, Oz B. Metastatic cardiac myxoma. Ann Thorac Surg 2001; 72: 623-5.

6. Al-Mateen M, Hood M, Trippel D, Insalaco SJ, Otto RK, Vitikainen KJ. Cerebral embolism from atrial myxoma in pediatric patients. Pediatrics 2003; 112: e162-7.

7. Ekinci EI, Donnan GA. Neurological manifestations of cardiac myxoma: a review of the literature and report of cases. Intern Med J 2004; 34: 243-9.

8. Dubernet J, Casanegra P, Chuaqui B, Maturana G, Braun S, Morán S et al. Myxomes auriculaires: Huit cas opérés dont quatre avec étude ultramicroscopique. Annales de Chirurgie 1981; 35 : 549-54.

9. Zalaquett R, Morán S, Irarrázaval MJ, Maturana G, Navarro M, Braun S et al. Acceso auricular izquierdo transeptal y superior combinado. Rev Chil Cirugía 1993; 45: 225-8.

10. Fang BR, Chiang CW, Hung JS, Lee YS, Chang CS. Cardiac myxoma: clinical experience in $24 \mathrm{pa}-$ tients. Int J Cardiol 1990; 29: 335-41.

11. Huston KA, Combs JJ JR, Lie JT, Giuliani ER. Left atrial myxoma simulating peripheral vasculitis. Mayo Clin Proc 1978; 53: 752-6.

12. Cohen AI, Mcintosh HD, Orgain ES. The mimetic nature of left atrialmyxomas: report of a case presenting as a severe systemic illness and simulating massive mitral insufficiency at cardiac catheterization. Am J Cardiol 1963; 11: 802-7.

13. Klaus R. Cardiac Myxomas. N Engl J Med 1995; 333; 24: 1610-17.

14. Saji T, Yanagawa E, Matsuura $\mathrm{H}$, Yamamoto $\mathrm{S}$, Ishikita $\mathrm{T}$, MATsuO $\mathrm{N}$ ET AL. Increased serum interleukin-6 in cardiac myxoma. Am Heart J 1991; 122: 579-80.

15. Seino Y, Ikeda U, Shimada K. Increased expression of interleukin 6 mRNA in cardiac myxomas. $\mathrm{Br}$ Heart J 1993; 69: 565-7.

16. Effert S, Domanig E. Diagnostik intraaurikulärer Tumoren und großer Thromben mit dem Ultraschall-Echoverfahren. Dtsch Med Wochenschr 1959; 84: 6-8.

17. Obeid Ai, Marvasti M, Parker F, Rosenberg J. Comparison of transthoracic and transesophageal echocardiography in diagnosis of left atrial myxoma. Am J Cardiol 1989; 63: 1006-8.
18. Mügge A, Daniel WG, Haverich A, Lichtlen PR. Diagnosis of noninfective cardiac mass lesions by two-dimensional echocardiography. Comparison of the transthoracic and transesophageal approaches. Circulation 1991; 83: 70-8.

19. Engberding R, Daniel WG, Erbel R, Kasper W, Lestuzzi C, Curtins JM et al. Diagnosis of heart tumours by transesophageal echocardiography: a multicentre study in 154 patients. Eur Heart J 1993; 14: 1223-8.

20. Ohshima H, Kawashima E, Ogawa Y, Tobise K, OnODERA S. Demonstration of the inner structure of a right atrial myxoma by transoesophageal echocardiography. Eur Heart J 1993; 14: 132-4.

21. Amano J, Kono T, Wada Y, Zhang T, Koide N, Fujimori M, Ito K. Cardiac myxoma: its origin and tumor characteristics. Ann Thorac Cardiovasc Surg 2003; 9: 215-21.

22. Crafoord C. Discussion of Glover RP. The technique of mitral commissurotomy. In: Lam CR, editor. Henry Ford Hospital: International Symposium on Cardiovascular Surgery. Philadelphia: Saunders, 1955; 202-3.

23. Thomas Ke, Winchel CP, Varco RL. Diagnostic and surgical aspects of left atrial tumors. J Thorac Cardiovasc Surg 1967; 53: 535-48.

24. Livi U, Bortolotti U, Milano A, Valente M, Prandi A, FrugOnI C ET AL. Cardiac myxomas: results of 14 years' experience. Thorac Cardiovasc Surg 1984; 32: 143-7.

25. Centofanti P, Rosa ed, Deorsola L, Actis Dato GM, Patane F, Torre ML et al. Primary cardiac tumors: Early and late results of surgical treatment in 91 patients. Ann Thorac Surg 1999; 68: 1236-41.

26. Bjessmo S, Ivert T. Cardiac myxoma: 40 years' experience in 63 patients. Ann Thorac Surg 1997; 63: 697-700.

27. Dein JR, Frist WH, Stinson EB, Miller DC, Baldwin JC, Oyer PE et Al. Primary cardiac neoplasms: Early and late results of surgical treatment in 42 patients. J Thorac Cardiovasc Surg 1987; 93: 50211.

28. Jones DR, Warden HE, Murray GF, Hill RC, Graeber GM, Cruzzavala JL et al. Biatrial approach to cardiac myxomas: A 30-year clinical experience. Ann Thorac Surg 1995; 59: 851-6.

29. Kamiya H, Yasuda T, Nagamine H, Sakakibara N, Nishida S, Kawasuji M, Watanabe G. Surgical treatment of primary cardiac tumors: 28 years' experience in Kanazawa University Hospital. Jpn Circ J 2001; 65: 315-9. 
30. Van Gelder HM, O'Brien DJ, Staples ED, Alexander JA. Familial cardiac myxoma. Ann Thorac Surg 1992; 53: 419-24.

31. Edwards A, Bermúdez C, Piwonka G, Berr ML, Zamorano J, Larraín E et al. Carney's syndrome: complex myxomas. Report of four cases and review of the literature. Cardiovasc Surg 2002; 10: 264-75.

32. SANDrini F, Stratakis C. Clinical and molecular genetics of Carney complex. Mol Genet Metab 2003; 78: 83-92.

33. Shinfeld A, Katsumata T, Westaby S. Recurrent cardiac myxoma: seeding or multifocal disease? Ann Thorac Surg 1998; 66: 285-8.

34. Bortolotti U, Scioti G, Guglielmi C, Milano A, Nardi C, Tartarini G. Recurrent myxoma of the left ventricle. Case report and review of the literature. J Cardiovasc Surg (Torino). 1999; 40: 233-5.

35. Becker P, Frangini S, Arnaiz P. Recurrent left atrial myxoma. Report of a case in a 2 year-old boy. Rev Méd Chile 2006; 134: 635-40.

36. Castells E, Ferrán V, Octavio de Toledo MC, Calbet JM, Benito M, Fontanillas C et al. Cardiac myxomas: surgical treatment, long-term results and recurrence. J Cardiovasc Surg (Torino) 1993; 34: 49-53.

37. Schaff HV, Mullany CJ. Surgery for cardiac myxomas. Semin Thorac Cardiovasc Surg 2000; 12: 77-88.

38. González R, Raffo M, Alarcón E, Gyhra A, Saldías R, Stockins A et al. Tumores cardiacos. Rev Chil Cirugía 2005; 57: 195-8. 